Article

\title{
The Flux of Phenolic Compounds through Silicone Membranes
}

\author{
John Prybylski and Kenneth B. Sloan* \\ Department of Medicinal Chemistry, University of Florida, P.O. Box 100485, Gainesville, \\ Florida 32610, USA; E-Mail: jprybylski.rx@ufl.edu
}

* Author to whom correspondence should be addressed; E-Mail: sloan@cop.ufl.edu;

Tel.: +1-352-273-7745; Fax: +1-352-392-9455.

Received: 11 July 2013; in revised form: 14 August 2013 / Accepted: 14 August 2013 /

Published: 21 August 2013

\begin{abstract}
Phenols as a class of molecules have been reported to exhibit higher log maximum fluxes through human stratum corneum, $\mathrm{SC}$, from water, $\log J_{\mathrm{MHAQ}}$, than other classes of molecules. This suggests that their corresponding log maximum fluxes through silicone from water, $\log J_{\mathrm{MPAQ}}$, may be useful to extend the existing $n=63 \log J_{\mathrm{MPAQ}}$ database to include more $\log J_{\mathrm{MPAQ}}$ values greater than 0.0 . The $\log J_{\mathrm{MPAQ}}$ values for $n=7$ phenols predicted to give $\log J_{\mathrm{MPAQ}}$ values greater than 0.0 based on their $\log J_{\mathrm{MHAQ}}$ values

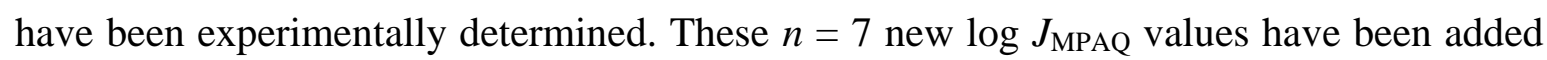
to the existing $n=63 \log J_{\text {MPAQ }}$ database to give a new $n=70$ database and the $n=7$ literature $\log J_{\text {MHAQ }}$ values have been added to the existing $n=48 \log J_{\text {MHAQ }}$ database (matched to the $n=63 \log J_{\mathrm{MPAQ}}$ database) to give a new $n=55$ database. The addition of the $n=7$ phenols improved the correlations of these flux databases when fitted to the Roberts-Sloan equation, RS, as well as the correlation between the matched experimental (Exp.) $\log J_{\text {MPAQ }}$ with the Exp. $\log J_{\text {MHAQ. }}$
\end{abstract}

Keywords: maximum flux; silicone membrane; Roberts-Sloan equation; water solubility; lipid solubility; human skin in vitro

\section{Introduction}

The rate-limiting barrier to diffusion of molecules through human skin is the stratum corneum, SC. The SC is comprised of highly dense, polar, proteinous corneocytes embedded in a lipid matrix. The lipid matrix in turn is comprised of multiple lipid bilayers containing mostly ceramides, fatty acids and 
cholesterol [1-3]. A tortuous pathway through this lipid matrix and around the corneocytes is generally considered to be the route followed by molecules diffusing through the SC. Since the route of molecules diffusing through the SC is lipid-like, the lipid solubility of molecules diffusing through the $\mathrm{SC}$ is an important physiochemical determinant of the efficiency of the diffusion process. Similarly, any surrogate for the SC must present with substantially lipid-like properties. Since silicone membranes present with highly lipid-like properties, it has been suggested that silicone membranes could be a surrogate for skin in diffusion cell studies and that those results could be used to predict diffusion through human skin [4,5]. Also, from a theoretical basis it can be assumed that, if the diffusion of unit mass per unit area per unit time, flux, through silicone membranes can be accurately modeled by an equation (Roberts-Sloan, RS, [6]) that accurately models maximum flux through human skin [7] exists, a linear relationship exists between the maximum flux of molecules through silicone membranes from water, $\log J_{\mathrm{MPAQ}}$, and their maximum flux through human skin in vitro from water, $\log J_{\text {MHAQ }}$ [5]. Thus experimental (Exp.) $\log J_{\text {MPAQ }}$ could be used to predict Exp. $\log J_{\text {MHAQ. }}$

The flux data for molecules from which the Roberts-Sloan, RS, equation was derived is based on the application of saturated solutions (suspensions of molecules in a solvent) to the membrane being used to give maximum flux, $J_{\mathrm{M}}$. Thus all the molecules are presented to the membrane at their maximum thermodynamic activity in that solvent [6-8]. Since the molecules are presented to the membrane at their maximum thermodynamic activity, at equilibrium the molecules are also at their maximum thermodynamic activity in the membrane [8,9], i.e., at their solubility limit or saturated solubility in the first few layers of the membrane, $S_{\mathrm{M} 1}$. Thus, $J_{\mathrm{M}}$ depends only on the solubility of the molecules in the first layers of the membrane, and unless the solvent changes the solubility limit of the membrane, the solvent/vehicle has no effect on $J_{\mathrm{M}}$. The form of the RS equation for predicting $J_{\mathrm{M}}$ derives from an expansion of Fick's law, Equation 1, so that the dependent variables are molecular weight, MW, solubility in a lipid, $S_{\mathrm{LIPID}}$, and solubility in water, $S_{\mathrm{AQ}} . S_{\mathrm{M} 1}$ can then be estimated from the product of the partition coefficient between the vehicle (water in this case, AQ) and a surrogate lipid for the membrane (octanol in this case, $\left.S_{\mathrm{OCT}}\right),\left(K_{\mathrm{OCT}: \mathrm{AQ}}\right)^{y} \cdot$ constant, and the solubility in the vehicle, $S_{\mathrm{AQ}}:\left(K_{\mathrm{OCT}: \mathrm{AQ}}\right)^{y} \cdot$ constant $S_{\mathrm{AQ}}$. Expansion of that product into solubilities and taking the logs gives: $\log S_{\mathrm{M} 1}=y \log S_{\mathrm{OCT}}-y \log S_{\mathrm{AQ}}+\log S_{\mathrm{AQ}}+\log$ constant $=y \log S_{\mathrm{OCT}}+(1-y) \log S_{\mathrm{AQ}}+\log$ constant.

$$
J=D / L\left(C_{\mathrm{M} 1}-C_{\mathrm{Mn}}\right): J_{\mathrm{M}}=D / L\left(S_{\mathrm{M} 1}-C_{\mathrm{Mn}}\right)
$$

where $D$ is the diffusion coefficient of the molecule in the membrane, $L$ is the thickness of the membrane, $C_{\mathrm{M} 1}$ is the concentration of the molecule in the first few layers of the membrane and $C_{\mathrm{Mn}}$ is the concentration in the last few layers of the membrane which is assumed to approach zero. A linear relationship must exist between $\log D+\log S_{\mathrm{M} 1}$ of molecules in a silicone membrane and $\log D+\log S_{\mathrm{M} 1}$ of molecules in human skin in order for a linear relationship between $\log J_{\mathrm{MPAQ}}$ and $\log J_{\mathrm{MHAQ}}$ to exist.

One problem with determining if the linear relationship between $\log J_{\mathrm{MPAQ}}$ and $\log J_{\mathrm{MHAQ}}$ exists is that there are only about $n=63$ molecules for which $\log J_{\text {MPAQ }}$ (output) and the necessary corresponding physicochemical properties ( $\log S_{\mathrm{AQ}}$ and $\log S_{\mathrm{OCT}}$, input) literature values exist which can be fitted to RS [5]. Of those $n=63$, only 18 molecules exhibit high output values; $\log J_{\text {MPAQ values }}$ greater than 0.0. Simple phenols present an opportunity to extend the existing $n=63 \log J_{\mathrm{MPAQ}}$ database to include more $\log J_{\mathrm{MPAQ}}$ values greater than 0.0 . The $\log J_{\mathrm{MHAQ}}$ values for $n=18$ phenols and their corresponding physicochemical properties that are necessary to determine their fit to RS were 
published by Roberts, et al. in 1977 [10]. The fit of the $n=18$ phenols to RS in the fit of the $n=62$ edited Flynn database to RS was published in 2007 [7]. In the edited Flynn database only $n=16$ of the $n=62$ molecules exhibited $\log J_{\mathrm{MHAQ}}$ values greater than 0.0 and of those $n=16, n=11$ were from among the $n=18$ phenol subset [10]. Thus, phenols as a subset represent molecules that exhibit physicochemical properties (input) that give higher flux (output) than other types of molecules give.

At present, only $n=6$ of the $n=18$ simple phenol subset from Roberts, et al. [10] have been included in the $n=63 \log J_{\text {MPAQ }}$ database and only $n=2$ exhibit $\log J_{\text {MPAQ }}$ greater than 0.0 . In order to improve the correlation of the $n=63 \log J_{\mathrm{MPAQ}}$ database with a matched $n=48 \log J_{\mathrm{MHAQ}}$ database, the number of $\log J_{\mathrm{MPAQ}}$ and $\log J_{\mathrm{MHAQ}}$ greater than 0.0 in each database should be increased. Hence, $n=7$ additional phenols have been selected from the $n=18$ subset which exhibit physicochemical properties (input) for which RS predicts high $\log J_{\text {MPAQ }}$ values (output). In addition, the $n=7$ phenols exhibit an average $\log J_{\mathrm{MHAQ}}$ value significantly greater than that of the $n=48 \log J_{\mathrm{MHAQ}}$ database: means $\pm 95 \%$ confidence intervals of $0.04 \pm 0.42 \log$ units and $-1.06 \pm 0.31 \log$ units, respectively. Given the increased range and total number of entries resulting from the addition of these $n=7$ compounds to the $n=48 \log J_{\mathrm{MHAQ}}$ database and the $n=63 \log J_{\mathrm{MPAQ}}$ database, the fit of these databases to the RS should improve, and correlation of the $\log J_{\text {MPAQ }}$ with $\log J_{\text {MHAQ values matched in }}$ these databases should also improve.

Further, since the addition of the $n=7$ new entries to the new $n=63 \log J_{\text {MPAQ }}$ database, each potentially exhibiting higher $\log J_{\mathrm{MPAQ}}$ values than the average of the initial $n=63 \log J_{\mathrm{MPAQ}}$ values, will change the relative distribution of flux values in the database, it is imperative to determine if other models would then fit the database better than they did before the addition of the $n=7$ new entries. Thus, we will also determine the fit of the new databases to the Kasting-Smith-Cooper (KSC) model [11] and to the Magnusson-Anissimov-Cross-Roberts (MACR) model [12] and compare these fits to the fit of RS to the new databases.

\section{Materials and Methods}

The phenolic compounds used are listed in Table 1. These compounds were obtained from Aldrich and their solubility values were acquired or approximated from literature sources. The phenols were all solids except for 3-methylphenol.

The measurement of maximum flux through silicone was performed according to a literature procedure [13] at $32{ }^{\circ} \mathrm{C}$, except that the silicone membrane was in contact with the receptor for only $24 \mathrm{~h}$ to condition them. The receptor was a $7.1 \mathrm{pH}$ phosphate buffer.

The donor suspensions were prepared by stirring approximately $0.5 \mathrm{~g}$ ( $1 \mathrm{~g}$ in the case of 3 -methylphenol) of the compounds in $10 \mathrm{~mL}$ of water for $24 \mathrm{~h}$. For all compounds, this surpassed the aqueous solubility by a factor of at least 20 , which ensured saturation and excess solid/oil present in the donor phase. After the membranes were conditioned, the receptor phases were changed and the donor suspensions (first application, $1 \mathrm{~mL}$ ) were applied; $n=3$. The donor cells were sealed by Parafilm. Samples were taken from the receptor every 2-3 h after application. Following sample collection, the receptor phases were changed to ensure sink conditions, and the donor suspensions were either changed or had more solid/oil added to the existing suspension, depending upon the visible extent of depletion. After 4-5 sampling intervals, the donor suspensions were removed with methanol 
and the receptors were changed. The membranes were leached with methanol in the donor phase for 48-72 $\mathrm{h}$ with samples taken and receptor phases changed every 12-24 h to remove any residual phenol in the membrane.

To ensure that flux data was not altered by possible membrane damage, a standard solute/solvent was applied and its flux determined. A donor suspension was prepared from $400 \mathrm{mg}$ of theophylline suspended with stirring in $6 \mathrm{~mL}$ of propylene glycol (PG) for $24 \mathrm{~h}$. This suspension (second application, $0.50 \mathrm{~mL}$ ) was applied to all the silicone membranes after they were leached with methanol (see above). Samples were taken from the receptor every $24 \mathrm{~h}$ after application for at least $72 \mathrm{~h}$ so that at least 3 samples were obtained. Following sample collection, the receptor phases were changed and the donor suspensions were changed every other sampling interval. After 3-5 sampling intervals, the diffusion cells were disassembled and the membranes were placed in a methanol bath for maintenance leaching.

The flux values of the first and second application were determined by UV absorption. The wavelengths $\left(\lambda_{\varepsilon}\right)$ and molar absorptivities $(\varepsilon)$ used for the phenolic compounds are listed in Table 1. The $\log$ flux of theophylline through silicone from PG, $\log J_{\mathrm{MPPG}}$, for each membrane was found to be within the standard deviation of the literature value of $-2.68 \pm 0.12 \log$ units [13].

Nonlinear regression was performed by SPSS 20.0 (Rel. 20.0.0). The compounds were fitted to the Roberts-Sloan equation for maximum flux, $\log J_{\mathrm{MAQ}}$ :

$$
\log J_{\mathrm{MAQ}}=x+y \log S_{\mathrm{OCT}}+(1-y) \log S_{\mathrm{AQ}}-z \mathrm{MW}
$$

to the KSC Equation:

$$
\log J_{\mathrm{MAQ}}=x+y \log S_{\mathrm{OCT}}-z \mathrm{MW}
$$

and to the MACR Equation:

$$
\log J_{\mathrm{MAQ}}=x-z \mathrm{MW}
$$

\section{Results and Discussion}

The results are displayed in Table 1 . All but 4-chloro-3,5-dimethylphenol exhibited a $\log J_{\mathrm{MPAQ}}$ greater than 0.0, and even it was very close. As a subset the $n=7$ simple phenols had an average $\log J_{\mathrm{MPAQ}}$ significantly greater than the average $\log J_{\mathrm{MPAQ}}$ of the $n=63 \log J_{\mathrm{MPAQ}}$ database: means \pm $95 \%$ confidence intervals $1.03 \pm 0.45 \log$ units and $-0.42 \pm 0.29 \log$ units, respectively. The average $\log J_{\mathrm{MPAQ}}$ in the $n=70 \log J_{\mathrm{MPAQ}}$ database has not significantly increased, but is no longer significantly less than 0.0 : mean $\pm 95 \%$ confidence interval $-0.27 \pm 0.29$. Unfortunately, the addition of the $n=7$ phenols did not significantly increase the average $\log J_{\text {MHAQ }}$ of the $n=55 \log J_{\text {MHAQ }}$ database relative to the $n=48 \log J_{\mathrm{MHAQ}}$ database: means $\pm 95 \%$ confidence intervals, $-0.918 \pm 0.29$ $\log$ units and $-1.058 \pm 0.31 \log$ units, respectively. 
Table 1. The relevant measured or literature physicochemical properties for the $n=7$ phenolic compounds used in this study.

\begin{tabular}{ccccccccc}
\hline Cmpd. $^{\mathbf{a}}$ & $\mathbf{M W}$ & $\mathbf{L o g} \boldsymbol{S}_{\mathbf{A Q}}{ }^{\mathbf{b}, \mathbf{d}}$ & $\mathbf{L o g} \boldsymbol{K}_{\mathbf{O C T}: \mathbf{A Q}}{ }^{\mathbf{b}}$ & $\mathbf{L o g} \boldsymbol{S}_{\mathbf{O C T}}{ }^{\mathbf{b}, \mathbf{d}}$ & $\boldsymbol{\lambda}_{\boldsymbol{\varepsilon}}{ }^{\mathbf{c}}$ & $\boldsymbol{\varepsilon}^{\mathbf{c}, \mathbf{e}}$ & $\mathbf{L o g} \boldsymbol{J}_{\mathbf{M P A Q}}{ }^{\mathbf{c}, \mathbf{f}}$ & $\mathbf{L o g} \boldsymbol{J}_{\mathbf{M H A Q}}{ }^{\mathbf{b}, \mathbf{f}}$ \\
\hline 1 & 143 & 1.55 & 3.10 & 4.65 & 283 & 1241 & 1.01 & 0.29 \\
2 & 157 & 0.28 & 3.39 & 3.67 & 285 & 1041 & -0.027 & -0.95 \\
3 & 122 & 1.61 & 2.35 & 3.96 & 277 & 1668 & 1.37 & 0.17 \\
4 & 108 & 2.29 & 1.95 & 4.24 & 276 & 1614 & 1.62 & 0.53 \\
5 & 163 & 1.49 & 3.08 & 4.57 & 285 & 1791 & 1.16 & 0.27 \\
6 & 197 & 0.66 & 3.69 & 4.35 & 312 & 4518 & 0.49 & -0.57 \\
7 & 108 & 2.36 & 1.96 & 4.32 & 271 & 1468 & 1.61 & 0.54 \\
\hline
\end{tabular}

${ }^{a}$ Substituted phenols. 1, 4-chloro-3-methyl; 2, 4-chloro-3,5-dimethyl; 3, 3,4-dimethyl; 4, 4-methyl; 5, 2,4-dichloro; 6, 2,4,6-trichloro; 7, 3-methyl; ${ }^{\text {b }}$ From Roberts et al. 1977 [10] and Majumdar et al. 2007 [7]; ${ }^{\mathrm{c}}$ Measured directly.

${ }^{\mathrm{d}}$ Solubility in water $\left(S_{\mathrm{AQ}}\right)$ or octanol $\left(S_{\mathrm{OCT}}\right)$ in $\mu$ mole $\mathrm{cm}^{-3}$; ${ }^{\mathrm{e}}$ Molar absorptivity coefficient in $\mathrm{L} \mathrm{mole}{ }^{-1} \mathrm{~cm}^{-1} ;{ }^{\mathrm{f}}$ Maximum flux through silicone $\left(J_{\mathrm{MPAQ}}\right)$ or human stratum corneum $\left(J_{\mathrm{MHAQ}}\right)$ from water in $\mu$ mole $\mathrm{cm}^{-2} \mathrm{~h}^{-1}$.

The addition of these $n=7$ phenols to the $n=63 \log J_{\text {MPAQ }}$ database and the $n=48 \log J_{\text {MHAQ }}$ database improved the fit of these databases to the RS as expected. The fit of the new $n=70 \log J_{\text {MPAQ }}$ database gave an $r^{2}$ of 0.907 , an average absolute residual $\log J_{\mathrm{MPAQ}}\left(\Delta \log J_{\mathrm{MPAQ}}\right)$ of $0.300 \log$ units and the coefficients $x=-1.606, y=0.695$ and $z=0.00490$ were all significant $(p<0.05)$ :

$$
\log J_{\mathrm{MPAQ}}=-1.606+0.695 \log S_{\mathrm{OCT}}+0.305 \log S_{\mathrm{AQ}}-0.00490 \mathrm{MW}
$$

The fit of the $n=70 \log J_{\mathrm{MPAQ}}$ database is an improvement over the $n=63 \log J_{\mathrm{MPAQ}}$ database, which had $r^{2}=0.896$ and $\Delta \log J_{\mathrm{MPAQ}}=0.310 \log$ units, but had similar coefficient values: $x=-1.607$, $y=0.701, z=0.00492$. The fit of the new $n=55 \log J_{\text {MHAQ }}$ database gave an $r^{2}$ of 0.883 , an average absolute residual $\log J_{\mathrm{MHAQ}}\left(\Delta \log J_{\mathrm{MHAQ}}\right)$ of $0.282 \log$ units and the coefficients $x=-3.005$ and $y=0.654$ were significant $(p<0.05)$, but the coefficient $z=0.00112$ was not significant $(p=0.25)$ :

$$
\log J_{\mathrm{MHAQ}}=-3.005+0.654 \log S_{\mathrm{OCT}}+0.346 \log S_{\mathrm{AQ}}-0.00112 \mathrm{MW}
$$

The lack of statistical significance for the $z$ coefficient indicates a need to further extend the $n=55$ $\log J_{\text {MHAQ }}$ database, since the significance of MW to maximum flux is well-established [12]. The fit of the $n=55 \log J_{\text {MHAQ }}$ database to the RS is an improvement over the $n=48 \log J_{\text {MHAQ }}$ database, which had $r^{2}=0.867$ and $\Delta \log J_{\mathrm{MHAQ}}=0.331 \log$ units and the coefficients $x=-2.763, y=0.635$ and $z=0.00207$. The $x$ and $y$ coefficients for the new $\log J_{\text {MHAQ }}$ database are substantially closer to those coefficients determined for the $n=62$ edited Flynn $\log J_{\text {MHAQ }}$ database: $x=-3.008, y=0.732$, $z=0.0048$. Relevant results are displayed in Table 2 and Figures 1 and 2. Figure 1 shows a plot of experimental (Exp.) $\log J_{\mathrm{MPAQ}}$ versus $\log J_{\mathrm{MPAQ}}$ calculated (Calc.) from the coefficients for the fit of the $n=70$ database to RS, and Figure 2 shows a plot of Exp. $\log J_{\text {MHAQ }}$ versus $\log J_{\text {MHAQ }}$ Calc. from the coefficients for the fit of the $n=55$ database to RS. 
Table 2. The calculated (Calc.), predicted (Pred.), and experimental (Exp.) maximum flux values through silicone from water $\left(\log J_{\mathrm{MPAQ}}\right)$ and through human stratum corneum from water $\left(\log J_{\mathrm{MHAQ}}\right)$ for the $n=7$ phenolic compounds.

\begin{tabular}{|c|c|c|c|c|c|c|}
\hline Cmpd. $^{a}$ & $\begin{array}{c}\text { Exp. } \\
\log J_{\text {MPAQ }}\end{array}$ & $\begin{array}{l}\text { Pred. } n=63 \\
\log J_{\mathrm{MPAQ}}\end{array}$ & $\begin{array}{l}\text { Calc. } n=70 \\
\log J_{\mathrm{MPAQ}}\end{array}$ & $\begin{array}{c}\text { Exp. } \\
\log J_{\text {MHAQ }}\end{array}$ & $\begin{array}{l}\text { Pred. } n=48 \\
\log J_{\mathrm{MHAQ}} \\
\text { b,e }\end{array}$ & $\begin{array}{l}\text { Calc. } n=55 \\
\log J_{\mathrm{MHAQ}}\end{array}$ \\
\hline 1 & 1.01 & 1.41 & 1.40 & 0.29 & 0.46 & 0.41 \\
\hline 2 & -0.027 & 0.28 & 0.26 & -0.95 & -0.66 & -0.68 \\
\hline 3 & 1.37 & 1.05 & 1.04 & 0.17 & 0.087 & 0.0053 \\
\hline 4 & 1.62 & 1.52 & 1.51 & 0.53 & 0.54 & 0.44 \\
\hline 5 & 1.16 & 1.24 & 1.23 & 0.27 & 0.35 & 0.32 \\
\hline 6 & 0.49 & 0.67 & 0.65 & -0.57 & -0.17 & -0.15 \\
\hline 7 & 1.61 & 1.60 & 1.59 & 0.54 & 0.62 & 0.52 \\
\hline$\Delta \log J_{\mathrm{MAQ}} \mathrm{g}$ & & 0.200 & 0.195 & & 0.159 & 0.162 \\
\hline
\end{tabular}

${ }^{a}$ Substituted phenols. 1, 4-chloro-3-methyl; 2, 4-chloro-3,5-dimethyl; 3, 3,4-dimethyl; 4, 4-methyl; 5, 2,4-dichloro; 6, 2,4,6-trichloro; 7, 3-methyl; ${ }^{\mathrm{b}}$ Given in units $\mu$ mole $\mathrm{cm}^{-2} \mathrm{~h}^{-1}$; ${ }^{\mathrm{c}}$ Using RS coefficients $x=-1.607, y=0.701$, $z=0.00492$ [6]; ${ }^{\mathrm{d}}$ Using RS coefficients $x=-1.606, y=0.695, z=0.00490 ;{ }^{\mathrm{e}}$ Using RS coefficients $x=-2.763, y=0.635$ and $z=0.00207$ [6]; ${ }^{\mathrm{f}}$ Using RS coefficients $x=-3.005, y=0.654, z=0.00112 ;{ }^{\mathrm{g}}$ Average absolute residual $\log J_{\mathrm{MPAQ}}$ or $\log J_{\text {MHAQ }}$ for the $n=7$ phenols.

Figure 1. The correlation of the calculated (Calc.) $\log J_{\mathrm{MPAQ}}$ from the fit of $n=70$ to RS with the experimental (Exp.) $\log J_{\mathrm{MPAQ}}$. The dashed lines represent the boundaries for residual $\log J_{\mathrm{MPAQ}}$ greater than 1.0 , the solid line indicates points where the Calc. $\log J_{\mathrm{MPAQ}}$ is equivalent to the Exp. $\log J_{\mathrm{MPAQ}}$. The filled circles indicate the $n=7$ phenols. The Calc. $\log J_{\mathrm{MPAQ}}$ values were determined with Equation 5: $\log J_{\mathrm{MPAQ}}=-1.606+0.695 \log S_{\mathrm{OCT}}+$ $0.305 \log S_{\mathrm{AQ}}-0.00490 \mathrm{MW} . r^{2}=0.907$, average absolute residual $\log J_{\mathrm{MPAQ}}=0.300$.

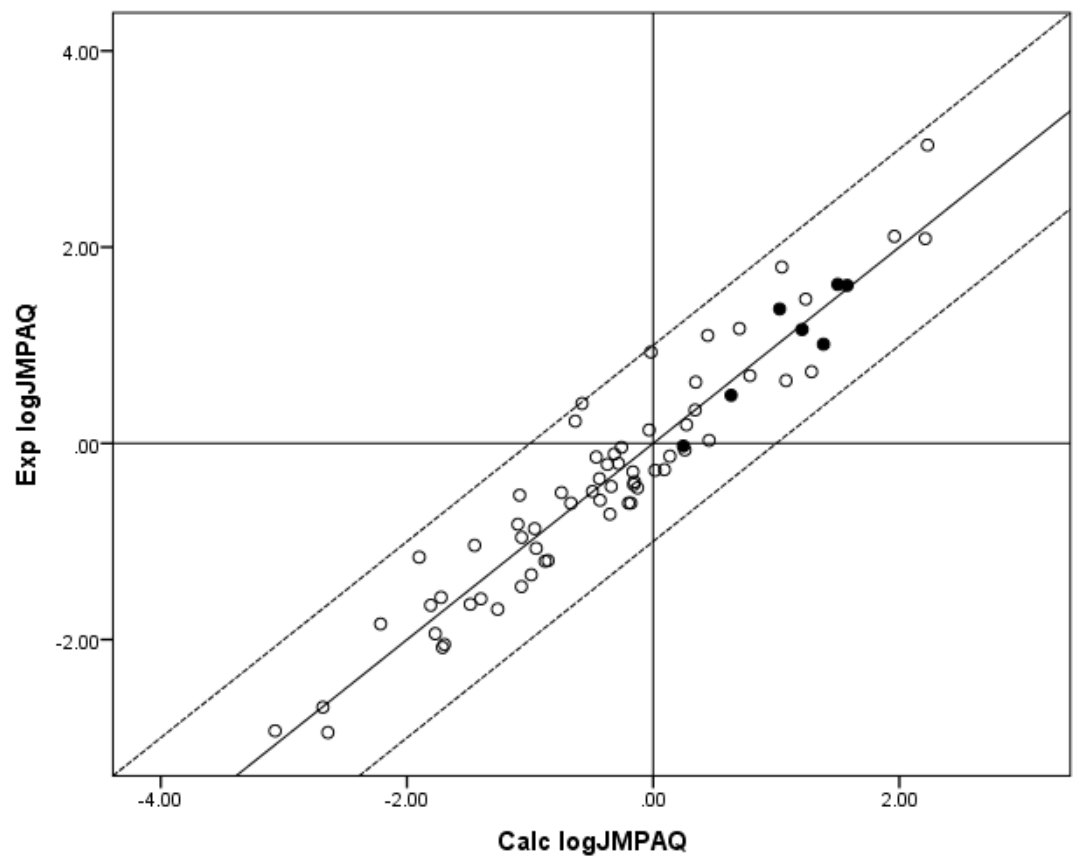


Figure 2. The correlation of the calculated (Calc.) $\log J_{\mathrm{MHAQ}}$ from the fit of $n=55$ to RS with the experimental (Exp.) log $J_{\mathrm{MHAQ}}$. The dashed lines represent the boundaries for residual Exp. $\log J_{\mathrm{MHAQ}}$ greater than 1.0, and the solid line indicates points where the Calc. $\log J_{\mathrm{MHAQ}}$ is equivalent to the Exp. $\log J_{\mathrm{MHAQ}}$. The filled circles indicate the $n=7$ phenols. The Calc. $\log J_{\mathrm{MHAQ}}$ values were determined with Equation 6: $\log J_{\mathrm{MHAQ}}=-3.005$ $+0.654 \log S_{\mathrm{OCT}}+0.346 \log S_{\mathrm{AQ}}-0.00112 \mathrm{MW}, r^{2}=0.883$, average absolute residual $\log J_{\mathrm{MHAQ}}=0.282$.

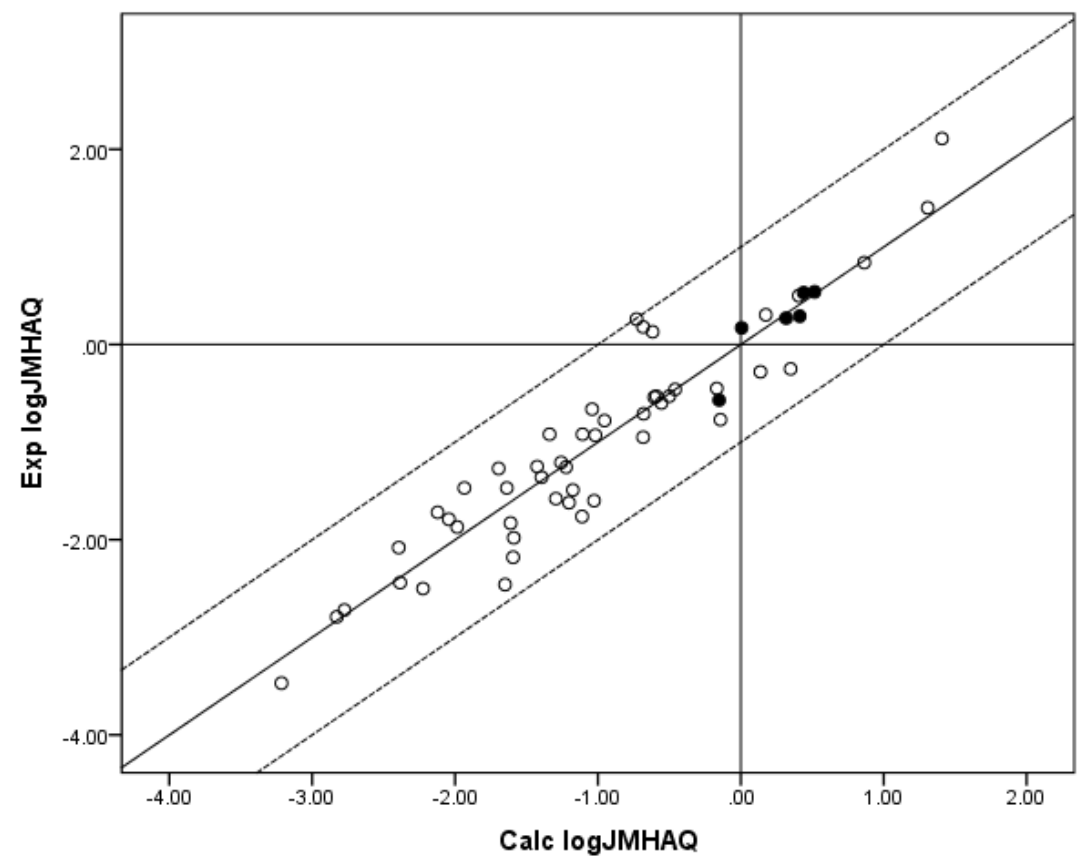

Plots of the individual independent variables, $\log S_{\mathrm{OCT}}, \log S_{\mathrm{AQ}}$ and $\mathrm{MW}$, against $\log J_{\mathrm{MPAQ}}(n=70)$ and against $\log J_{\mathrm{MHAQ}}(n=55)$ gave the following $r^{2}$ values: (a) $\log J_{\mathrm{MPAQ}}$ versus $\log S_{\mathrm{OCT}}, r^{2}=0.677$; versus $\log S_{\mathrm{AQ}}, r^{2}=0.554$; versus $\mathrm{MW}, r^{2}=0.541$; (b) $\log J_{\mathrm{MHAQ}}$ versus $\log S_{\mathrm{OCT}}, r^{2}=0.603$; versus $\log S_{\mathrm{AQ}}, r^{2}=0.526$; versus $\mathrm{MW}, r^{2}=0.520$. All regression equations had statistically significant $(p<0.05)$ slope and intercept estimates. In each case, the best regression of the individual independent

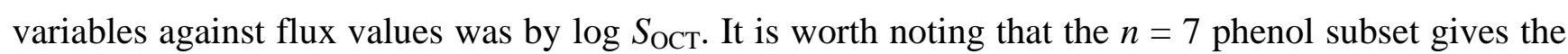
following $r^{2}$ values and significance profiles when the individual independent variables $\log S_{\mathrm{OCT}}$ and $\log S_{\mathrm{AQ}}$ are plotted against $\log J_{\mathrm{MPAQ}}$ and $\log J_{\mathrm{MHAQ}}$ : (a) $\log J_{\mathrm{MPAQ}}$ versus $\log S_{\mathrm{OCT}}, r^{2}=0.180$, without a statistically significant slope $(p=0.34)$ or intercept $(p=0.51)$; versus $\log S_{\mathrm{AQ}}, r^{2}=0.949$, with a statistically significant slope $(p<0.05)$, but without a statistically significant intercept $(p=0.50)$; (b) $\log J_{\mathrm{MHAQ}}$ versus $\log S_{\mathrm{OCT}}, r^{2}=0.335$, without a statistically significant slope $(p=0.17)$ or intercept ( $p=0.18)$; versus $\log S_{\mathrm{AQ}}, r^{2}=0.933$, with statistically significant $(p<0.05)$ slope and intercept. The improved dependence of maximum flux from water on the aqueous solubility of highly water-soluble compounds is demonstrated here as a property of both silicone and human stratum corneum, and will be a topic of future investigations. When two individual independent variables, $\log S_{\mathrm{OCT}}$ and $\mathrm{MW}$, from the $n=70 \log J_{\mathrm{MPAQ}}$ database were fitted to the KSC equation (Equation 3) the following $x, y$ and $z$ coefficients to the parameters were obtained along with $r^{2}$ and $\Delta \log J_{\mathrm{MPAQ}}$ values: $x=-0.923$, 
$y=0.794, z=0.0089, r^{2}=0.797$ and $\Delta \log J_{\mathrm{MPAQ}}=0.431$. All but the $y$ coefficient $(p=0.069)$ were statistically significant $(p<0.05)$ :

$$
\log J_{\text {MPAQ }}=-0.923+0.794 \log \mathrm{S}_{\mathrm{OCT}}-0.0089 \mathrm{MW}
$$

Figure 3 shows a plot of Exp. $\log J_{\mathrm{MPAQ}}$ versus $\log J_{\mathrm{MPAQ}}$ values calculated from the coefficients for the fit of the $n=70$ database to Equation 7. Although the $r^{2}$ was substantially improved by including MW with $\log S_{\mathrm{OCT}}$ as independent variables in the regression against flux, the $r^{2}$ was poorer than the $r^{2}$ for the fit of all three independent variables to the Roberts-Sloan Equation (Equation 5). Similarly, when two individual independent variables, $\log S_{\mathrm{OCT}}$ and $\mathrm{MW}$, from the $n=55 \log J_{\mathrm{MHAQ}}$ database were fit to the KSC equation (Equation 8) the following $x, y$ and $z$ coefficients to the parameters were obtained along with $r^{2}$ and the $\Delta \log J_{\mathrm{MHAQ}}$ values: $x=-1.252, y=0.602, z=0.0080, r^{2}=0.723$ and $\Delta \log J_{\mathrm{MHAQ}}=0.441$. The estimates for the coefficients were all statistically significant $(p<0.05)$ :

$$
\log J_{\text {MHAQ }}=-1.252+0.602 \log S_{\text {OCT }}-0.0080 \mathrm{MW}
$$

Figure 3. The correlation of the calculated (Calc.) $\log J_{\mathrm{MPAQ}}$ from the fit of $n=70$ to KSC with the experimental (Exp.) $\log J_{\mathrm{MPAQ}}$. The dashed lines represent the boundaries for residual Exp. $\log J_{\mathrm{MPAQ}}$ greater than 1.0, the solid line indicates points where the Calc. $\log J_{\mathrm{MPAQ}}$ is equivalent to the Exp. $\log J_{\mathrm{MPAQ}}$. The filled circles indicate the $n=7$ phenols. The Calc. $\log J_{\text {MPAQ }}$ values were determined with Equation 7: $\log J_{\text {MPAQ }}=-0.923+$ $0.794 \log S_{\text {OCT }}-0.0089 \mathrm{MW}, r^{2}=0.797$, average absolute residual $\log J_{\mathrm{MPAQ}}=0.431$.

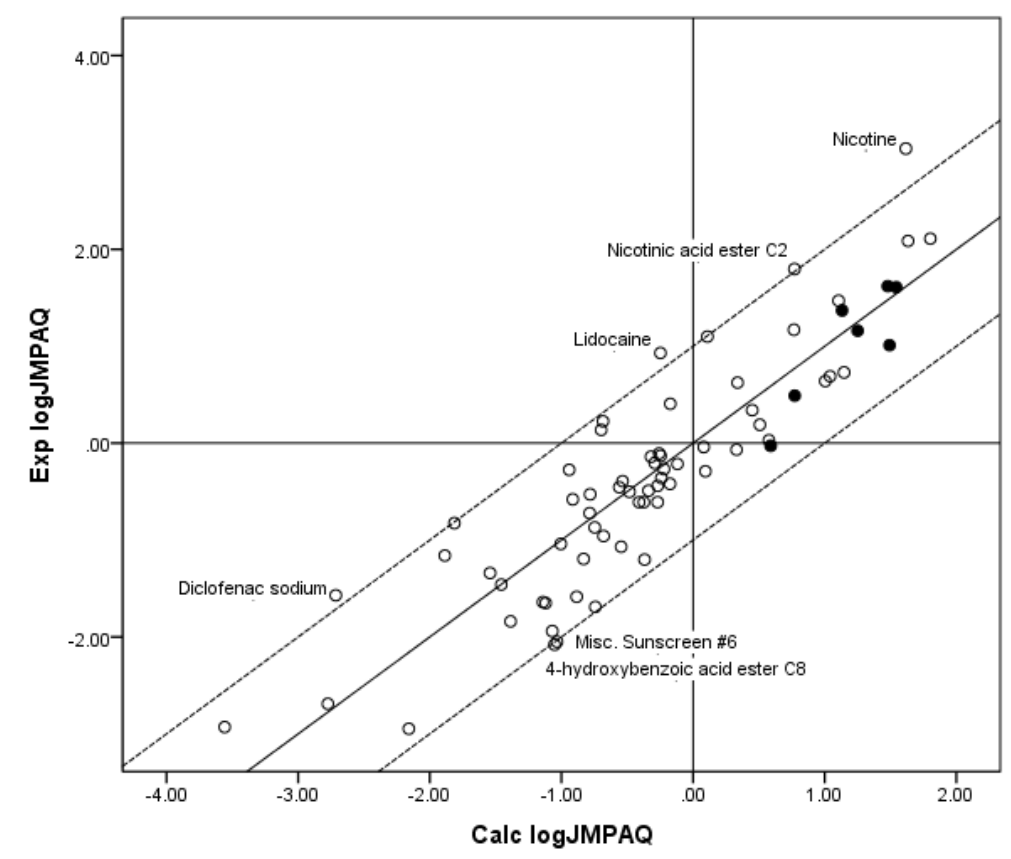

Figure 4 shows a plot of Exp. $\log J_{\mathrm{MHAQ}}$ versus $\log J_{\mathrm{MHAQ}}$ values calculated from the coefficients for the fit of the $n=55$ database to Equation 8. Again, although a substantial improvement in $r^{2}$ was obtained by including MW with $\log S_{\mathrm{OCT}}$ as independent variables in the regression against flux, the $r^{2}$ was poorer than the $r^{2}$ for the fit of all three independent variables to the RS equation (Equation 6). The fit of both databases to the MACR equation (Equation 4), which is the remaining model used to predict maximum flux, is simply the regression of MW against $\log J_{\mathrm{MPAQ}}$ or $\log J_{\mathrm{MHAQ}}$ shown above to 
give a somewhat poorer fit than regression of the two individual independent variables, $\log S_{\mathrm{OCT}}$ or $\log S_{\mathrm{AQ}}$, against $\log J_{\mathrm{MPAQ}}$ or $\log J_{\mathrm{MHAQ}}$. Finally, it should be noted the popular Potts-Guy Equation [14] was not included as a model because its output is permeability coefficient which is not clinically relevant.

Figure 4. The correlation of the calculated (Calc.) $\log J_{\mathrm{MHAQ}}$ from the fit of $n=55$ to KSC with the experimental (Exp.) log $J_{\mathrm{MHAQ}}$. The dashed lines represent the boundaries for residual Exp. $\log J_{\mathrm{MHAQ}}$ greater than 1.0, and the solid line indicates points where the Calc.

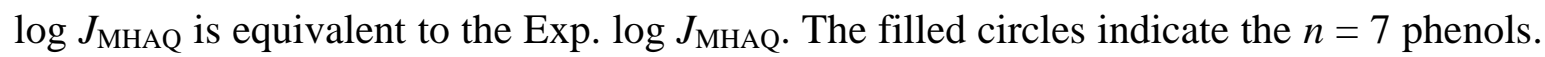
The Calc. $\log J_{\text {MHAQ }}$ values were determined with Equation 8: $\log J_{\text {MHAQ }}=-1.252+$ $0.602 \log S_{\mathrm{OCT}}-0.0080 \mathrm{MW}, r^{2}=0.723$, average absolute residual $\log J_{\mathrm{MHAQ}}=0.441$.

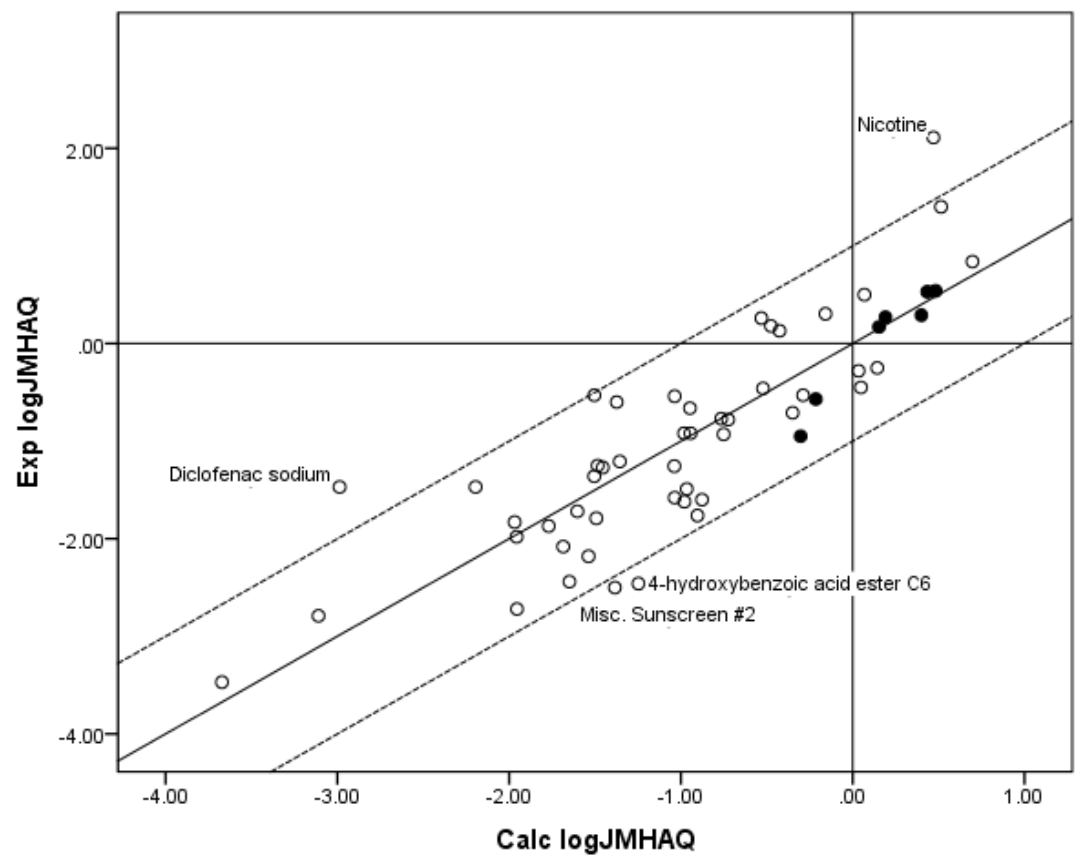

The new $n=52$ database of compounds contributing to both the $n=70 \log J_{\mathrm{MPAQ}}$ database and the $n=55 \log J_{\mathrm{MHAQ}}$ database also gives a higher correlation between Exp. $\log J_{\mathrm{MPAQ}}$ and Exp. $\log J_{\mathrm{MHAQ}}$ than the previous $n=45$ database. A linear regression yielded the expression Exp. $\log J_{\mathrm{MHAQ}}=0.859$ Exp. $\log J_{\mathrm{MPAQ}}-0.837, r^{2}=0.856$, which is an improvement over $r^{2}=0.838$ for the $n=45$ database. Figure 5 shows the plot of Exp. $\log J_{\mathrm{MPAQ}}$ versus Exp. $\log J_{\mathrm{MHAQ}}$ for $n=52$. 
Figure 5. The correlation of the $n=52 \log J_{\mathrm{MHAQ}}$ with $\log J_{\mathrm{MPAQ}}$ database. The dashed lines represent the boundaries for residual Exp. $\log J_{\mathrm{MHAQ}}$ greater than 1.0, and the solid line is the regression equation. The filled circles indicate the $n=7$ phenols. The regression information is in the figure.

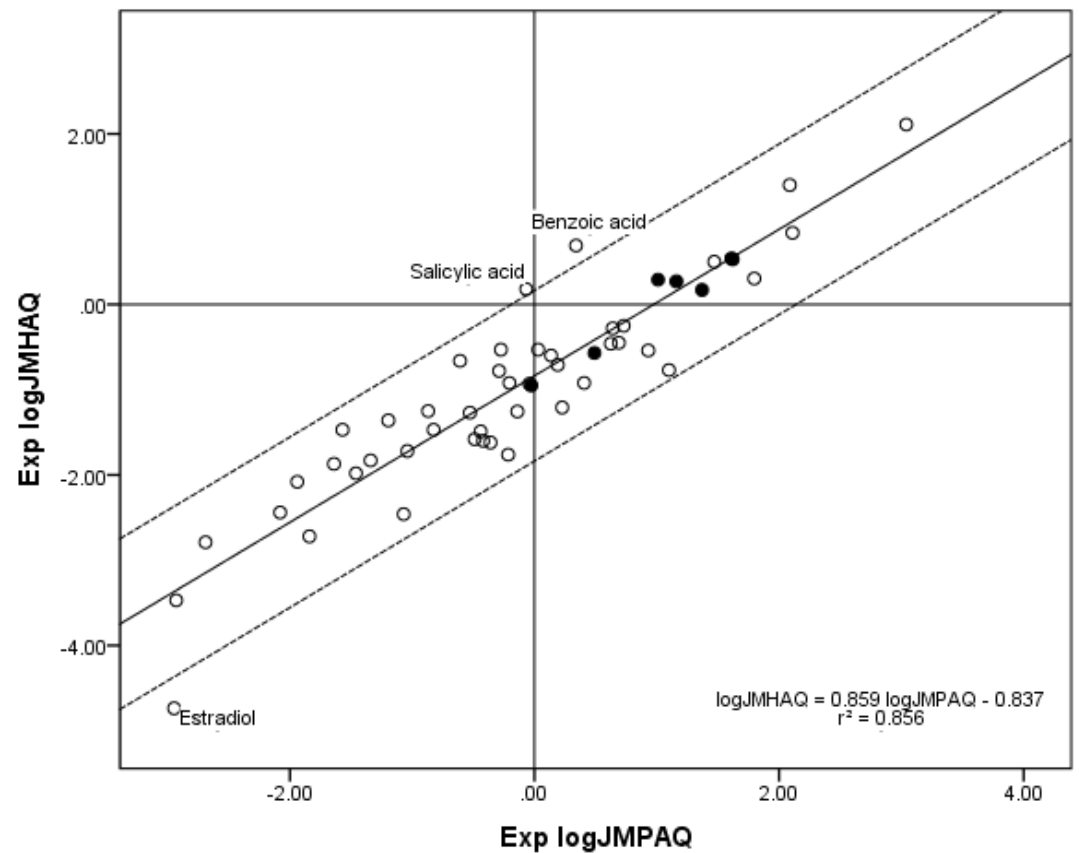

\section{Conclusions}

The addition of the $n=7$ phenols improved all aspects of the $\log J_{\mathrm{MPAQ}}$ database with regards to comparison with the matched $\log J_{\mathrm{MHAQ}}$ database. Along with strengthening the validity of the assertion that silicone membranes are good surrogates for human stratum corneum, these improvements demonstrate that this surrogate nature holds for a wider range of $\log J_{\mathrm{MPAQ}}$ and $\log J_{\mathrm{MHAQ}}$ values than had been previously reported.

\section{Conflicts of Interest}

The authors declare no conflict of interest.

\section{References}

1. Downing, D.T. Lipid and protein structures in the permeability barrier of mammalian epidermis. J. Lipid Res. 1992, 33, 301-313.

2. Bouwstra, J.A.; Gooris, G.S.; Dubbelaar, F.E.; Ponec, M. Phase behavior of stratum corneum lipid mixtures based on human ceramides: The role of natural and synthetic ceramide 1. J. Investig. Dermatol. 2002, 18, 606-617.

3. Bouwstra, J.A.; Gooris, G.S.; van der Spek, J.A.; Bras, W. Structural investigations of human stratum corneum by small-angle X-ray scattering. J. Investig. Dermatol. 1991, 97, 1005-1012. 
4. Wasdo, S.C.; Juntunen, J.; Devarajan, H.; Sloan, K.B. A correlation of flux through a silicone membrane with flux through hairless mouse skin and human skin in vitro. Int. J. Pharm. 2009, $373,62-67$.

5. Sloan, K.B.; Synovec, J.; Ketha, H. A surrogate for topical delivery in human skin: Silicone membranes. Ther. Deliv. 2013, 4, 203-224.

6. Roberts, W.J.; Sloan, K.B. Correlation of aqueous and lipid solubilities with flux for prodrugs of 5-fluorouracil, theophylline, and 6-mercaptopurine: A Potts-Guy approach. J. Pharm. Sci. 1999, 88, 515-522.

7. Majumdar, S.; Thomas, J.; Wasdo, S.; Sloan, K.B. The effect of water solubility of solutes on their flux through human skin in vitro. Int. J. Pharm. 2007, 329, 25-36.

8. Higuchi, T. Physical chemical analysis of percutaneous absorption process from creams and ointments. J. Soc. Cosmet. Chem. 1960, 11, 85-97.

9. Sloan, K.B.; Wasdo, S.C.; Rautio, J. Design for optimized topical delivery: Prodrugs and paradigm change. Pharm. Res. 2006, 23, 2729-2747.

10. Roberts, M.S.; Anderson, R.A.; Swarbrick, J. Permeability of human epidermis to phenolic compounds. J. Pharm. Pharmacol. 1977, 29, 677-683.

11. Kasting, G.B.; Smith, R.L.; Cooper, E.R. Effect of Lipid Solubility and Molecular Size on Percutaneous Absorption. In Pharmacology and the Skin; Shroot, B., Schaefer, H., Eds.; Karger: Basel, Switzerland, 1987; Volume 1, pp. 138-153.

12. Magnusson, B.M.; Anissimov, Y.G.; Cross, S.E.; Roberts, M.S. Molecular size as the main determinant of solute maximum flux across the skin. J. Investig. Dermatol. 2004, 122, 993-999.

13. Wasdo, S.; Juntunen, J.; Devarajan, H.; Murray, T.; Nickels, D.; Singh, S.; Shanks, T.; Ulmer, K.; Sloan, K.B. Modeling of flux through silicone membranes from water. Eur. J. Pharm. Sci. 2008, 34, 321-332.

14. Potts, R.O.; Guy, R.H. Predicting skin permeability. Pharm. Res. 1992, 9, 663-669.

(C) 2013 by the authors; licensee MDPI, Basel, Switzerland. This article is an open access article distributed under the terms and conditions of the Creative Commons Attribution license (http://creativecommons.org/licenses/by/3.0/). 